\title{
Atuação fisioterápica na capacidade funcional do idoso institucionalizado
}

\author{
Jaqueline Colombo Ely*; Aline Fernandes Fermino**; Gabriela Rech Bassani**; \\ Henrique Sulzbach de Oliveira**; Juliane Walter**; Sandra Helena Estevão do Amaral ${ }^{\star *}$; Adriana Arnt Brito ${ }^{* \star *}$
}

\section{Resumo}

A dependência funcional vem se destacando como um importante problema de saúde pública. A fisioterapia é essencial para uma atenção ampla da saúde do idoso institucionalizado. A atuação fisioterápica pode ser realizada no âmbito da atenção primária, secundária ou terciária à saúde. O objetivo desta revisão bibliográfica foi descrever os argumentos da literatura sobre a atuação da fisioterapia na capacidade funcional de idosos institucionalizados. Este estudo de revisão abordou estudos publicados nos bancos de dados eletrônicos Scielo e Bireme e acervo bibliográfico do Centro Universitário Univates.

Palavras-chave: Idoso. Instituição de longa permanência para idosos. Fisioterapia.

\section{Introdução}

O envelhecimento populacional, antes restrito aos países desenvolvidos, tornou-se realidade em nosso meio. $\mathrm{O}$ crescimento desta população, em números absolutos e relativos, é um fenômeno mundial e está ocorrendo de forma sem precedentes. De acordo com a última Pesquisa Nacional por Amostra de Domicílios (PNAD, 2006), a população de idosos ultrapassa 19 milhões, correspondendo a aproximadamente $13 \%$ da população brasileira. (IBGE, 2004).

A preocupação em relação à capacidade funcional vem emergindo como destaque no campo da geriatria e gerontologia, pelo fato de que a dependência funcional tende a se tornar um problema de saúde pública. (CALDAS, 2003). A capacidade funcional dos idosos deve ser destacada como um processo dinâmico, visto que sua evolução pode ser modifi-

* Fisioterapeuta pelo Centro de Ciências Biológicas e da Saúde do Centro Universitário Univates, Lajeado RS. Mestranda do Programa de Pós-Graduação em Envelhecimento Humano pela Universidade de Passo Fundo. Endereço para correspondência: Jaqueline Colombo Ely, Rua Emílio Conrad, 585, CEP 95900-000, Lajeado - RS. E-mail: jcolomboely@yahoo.com.br.

** Fisioterapeutas pelo Centro de Ciências Biológicas e da Saúde do Centro Universitário Univates, Lajeado - RS.

**** Fisioterapeuta especialista em Ortopedia e Traumatologia. Mestra em Desenvolvimento Regional pela Universidade de Santa Cruz do Sul. Professora e supervisora da disciplina de Estágio Supervisionado em Saúde Coletiva II do Centro de Ciências Biológicas e da Saúde, do Centro Universitário Univates.

$\hookrightarrow$ Recebido em agosto de 2008 - Aceito em março de 2009.

$\rightarrow$ doi:10.5335/rbceh.2009.028 
cada ou até mesmo prevenida se houver assistência adequada. A manutenção da capacidade funcional representa um novo paradigma para a saúde, particularmente relevante para o idoso, já que para ele os níveis de funcionalidade e independência são questões mais relevantes do que somente a presença de condições mórbidas. (GURALNIK et al., 1989). A fisioterapia geriátrica tem como objetivo principal a independência do idoso para as tarefas básicas de atividade de vida diária, no anseio de minimizar as consequências do processo de senescência e senilidade.

\section{Desenvolvimento}

O incremento no número de idosos se associa, em diversos países, ao aumento da necessidade por instituições de longa permanência. (CHAIMOWICZ; GRECO, 1999). Idosos residentes na comunidade nas principais capitais brasileiras apresentam alta prevalência de fatores de risco para a institucionalização: doenças crônico-degenerativas e suas sequelas, hospitalização recente e dependência para realizar atividades de vida diária. Além desses, fatores como morar só, suporte social precário e baixa renda são cada vez mais frequentes no Brasil. (CHAIMOWICZ, 1997).

O objetivo do presente trabalho é descrever a atuação da fisioterapia na capacidade funcional de idosos institucionalizados.

Estudos mostram que a diminuição da capacidade funcional limita a independência do idoso na execução das atividades de vida diária, reduz a qualidade de vida e confere altos riscos para mortalidade, dependência, institucionalização, hospitalização e cuidados. (FIRED; GURALNIK, 1997). Os fatores mais fortemente associados com a incapacidade funcional estão relacionados com a presença de algumas doenças, deficiências ou problemas médicos. (ROSA et al., 2003). Sabe-se que tanto doenças físicas quanto mentais podem levar à dependência, à diminuição da capacidade funcional e até mesmo à perda da autonomia. (VERAS, 2002).

Além disso, convém ressaltar que há maior prevalência de incapacidade e dependência funcional em idosos do sexo feminino, forte determinante de dependência. (FIRED; GURALNIK, 1997). Esse aspecto está intimamente relacionado à redução da massa muscular decorrente do envelhecimento, mesmo em idosas sem doenças crônicas. (SILVA et al., 2006). Além deste, o declínio fisiológico de outros componentes da função motora constitui um dos fatores mais significativos da dependência funcional. (GUCCIONE, 1992). Mesmo em idosas sem doenças prévias, o fato de apresentarem maior expectativa de vida corrobora a maior possibilidade de desenvolver doenças crônicas que incapacitam ou auxiliam na diminuição da capacidade funcional. (ROSA et al., 2003).

$\mathrm{Na}$ geriatria, a principal meta no cuidado é a manutenção da independência e da autonomia do idoso. (DIOGO; NERI; CACHIONI, 2004). A manutenção da qualidade de vida está intimamente vinculada à autonomia e independência, os quais são bons indicadores de saúde para a população idosa. (NETTO, 2002). Capacidade funcional surge, portanto, 
como um novo paradigma para a saúde, particularmente relevante para o idoso (FILLENBAUM, 1984), já que para ele os níveis de funcionalidade e independência são questões mais importantes do que somente a presença de condições mórbidas. (GURALNIK et al., 1989). De acordo com a portaria $n^{\circ} 1395 / \mathrm{GM}$, que dispõe sobre a Política Nacional de Saúde do Idoso (BRASIL, 1999), capacidade funcional é definida como a capacidade de manter as habilidades físicas e mentais necessárias para uma vida independente e autônoma.

Em geriatria, a avaliação da capacidade funcional é imprescindível. Define-se avaliação funcional como a observação e mensuração da capacidade de realização das tarefas básicas de vida diária. É geralmente usada, num sentido mais restrito, para se referir à medida de habilidade de uma pessoa para cumprir com suas responsabilidades diárias e desempenhar as tarefas de autocuidado (DIOGO; NERI; CACHIONI, 2004).

Um modelo de atenção à saúde centrado na avaliação da capacidade funcional deve ser, portanto, o exemplo a ser praticado em relação à atenção à saúde do idoso. Nesse contexto, o estabelecimento de prioridades e ações de saúde em geriatria deve nortear as novas políticas de saúde. (VERAS, 2002). A fisioterapia é a profissão da área da saúde que aplica e utiliza medidas de avaliação da capacidade funcional de forma mais importante. Medidas de avaliação da capacidade funcional têm sido amplamente utilizadas em pesquisas epidemiológicas sobre envelhecimento e na avaliação individual do paciente idoso. (DIOGO;
NERI; CACHIONI, 2004).

Além de poder avaliar um grande espectro de habilidades e predizer alguns achados, como quedas, admissão em instituições de cuidado e risco de morte, uma das principais razões para a grande utilização de medidas de desempenho funcional é que, por se tratar de métodos objetivos e padronizados de avaliação geriátrica, oferece mais subsídios para instrumentalizar e operacionalizar a atenção à saúde do idoso, revelar necessidades e determinar a utilização de recursos. (DIOGO; NERI; CACHIONI, 2004). Ainda, as informações geradas pela avaliação da capacidade funcional possibilitam conhecer o perfil dos idosos através da utilização de uma ferramenta simples e útil, o que pode auxiliar na definição de estratégias de promoção de saúde para os idosos, visando retardar ou prevenir as incapacidades. (LEE, 2000).

O envelhecimento saudável é "resultante da interação multidimensional entre saúde física, saúde mental, independência na vida diária, integração social, suporte familiar e independência econômica" (RAMOS, 2003) e é baseado na capacidade funcional. Cabe ao profissional fisioterapeuta que atua em geriatria e gerontologia atentar para a questão multifatorial do envelhecimento, tendo, portanto, uma visão abrangente. A fisioterapia é uma das profissões da área da saúde imprescindível para uma atenção ampla da saúde do idoso no sistema de saúde. A atuação profissional pode ser realizada nos âmbitos da atenção primária, secundária ou terciária à saúde. 
As alterações morfológicas, estruturais e funcionais próprias do processo de senescência, associadas à maior prevalência de doenças crônicas no processo de senilidade, podem levar à deterioração da habilidade de manutenção da independência. Por outro lado, é necessário ressaltar que mesmo um portador de doença poderá se sentir saudável, ativo em seu meio e ter, em última análise, boa qualidade de vida. (NETTO, 2002). Assim, a saúde do idoso não é mais medida pela presença ou não de doenças, mas pelo grau de preservação da capacidade funcional. Cabe ao profissional fisioterapeuta preservar, manter, desenvolver ou restaurar a integridade físico-funcional dos indivíduos. (SULLIVAN; SCHMITZ, 2004). Ações preventivas, assistenciais e de reabilitação devem objetivar a melhoria da capacidade funcional ou a manutenção da mesma e, sempre que possível, sua recuperação. (DIOGO, NERI; CACHIONI, 2004).

A fisioterapia geriátrica tem como objetivo principal a independência do idoso para as tarefas básicas de atividade de vida diária, no anseio de minimizar as consequências das alterações fisiológicas e patológicas do envelhecimento, garantindo a melhoria da mobilidade e favorecendo uma qualidade de vida satisfatória, que é julgada pelo idoso mais pelo nível funcional e grau de independência do que pela presença de limitações específicas e isoladas. (DIOGO; NERI; CACHIONI, 2004). Além disso, a atuação fisioterápica representa uma estratégia elementar para contribuir para um estilo de vida mais ativo, proporcionando um envelhecer motor $\mathrm{e}$ funcional com saúde, qualidade e maior independência funcional possível. (PEREIRA et al., 2002).

\section{Conclusão}

Com o estudo observou-se que ações fisioterápicas de prevenção, reabilitação, manutenção e apoio devem se centrar na manutenção da capacidade funcional ou na melhoria desta e, sempre que possível, sua recuperação. É um enfoque que transcende o simples tratamento de doenças específicas, uma vez que o conceito de saúde se inclina para a manutenção da independência e autonomia, não envolvendo somente a ausência de doenças crônicas. A atenção à saúde do idoso deve, portanto, ter como objetivo maior a manutenção da máxima capacidade funcional do indivíduo que envelhece. Isso remete à valorização da independência e autonomia do idoso e à conservação de sua máxima capacidade físico-funcional e mental.

Physiotherapeutic action in institutionalizaded old adults's functional capacity

\section{Abstract}

Functional dependence has been considered as an important public health issue. Physioptherapy is an occupation essential to wide attention in institutionalizaded old adults. The physiotherapic action can be done at primary, secondary and tertiary health care. The aim of this review was describe studies about physiotherapic action in functional capacity of old adults living in a long-permanence institution to elderly. This revision study approached studies published using electronic database Scielo 
and Bireme and bibliographical collection of Centro Universitário Univates.

Key words: Elderly. Homes for the Aged. Physiotherapy.

\section{Referências}

BRASIL. Portaria 1.395/GM, de 10 de dezembro de 1999. Política de Saúde do Idoso. Disponível em: <www.ufrgs.br/3idade/portaria1395gm.html>. Acesso em: 14 jun. 2008.

CALDAS, C. P. Envelhecimento com dependência: responsabilidades e demandas da família. Cadernos Saúde Pública, Rio de Janeiro, v. 19, n. 3, p. 773-781, 2003.

CHAIMOWICZ, F. A saúde dos idosos brasileiros às vésperas do século XXI: problemas, projeções e alternativas. Revista de Saúde Pública, São Paulo, v. 31, n. 2, p. 184-200, 1997.

CHAIMOWICZ, F.; GRECO, D. B. Dinâmica da institucionalização de idosos em Belo Horizonte, Brasil. Revista de Saúde Pública, São Paulo, v. 33, n. 5, p. 454-460, 1999.

DIOGO, M. J. D.; NERI, A. L.; CACHIONI M. Saúde e qualidade de vida na velhice. São Paulo: Alínea, 2004.

FILLENBAUM, G. G. The wellbeing of the elderly: aproaches to the multidimensional assessment. Geneva: World Health Organization, 1984.

FIRED, L. P.; GURALNIK, J. M. Disability in older adults: evidence regarding significance, etiology and risk. Journal American Geriatrics Society; v. 45, n. 1, p. 92-100, 1997.

GUCCIONE, A. A Functional assessment of the elderly. In: GUCCIONE, A. A. (Ed.). Geriatric plysical therapy. Boston: Mosby, 1992. p. 921-928.

GURALNIK, J. M. et al. Physical performance measures in aging research. J. Gerontol., v. 44, n. 5, p. 141-146, 1989.

INSTITUTO BRASILEIRO DE GEOGRAFIA E ESTATÍSTICA. Projeção da População do Brasil por sexo e idade para o período de 1980-2050 - Revisão 2004. Metodologia e Resultados. Disponível em: <www.ibge.gov. br>. Acesso em: 14 jun. 2008.

LEE, Y. The predictive value of self assessed general, physical, and mental health on functional decline and mortality in older adults. J. Epidemiol Community Health, v. 54, p. 123-129, 2000.

NETTO, M. P. Gerontologia: a velhice e o envelhecimento em visão globalizada. São Paulo: Atheneu, 2002.

PEREIRA, L. S. M. et al. Fisioterapia. In: FREITAS E. V. et al. (Org.). Tratado de geriatria e gerontologia. Rio de Janeiro: Guanabara Koogan; 2002.

RAMOS, L. R. Fatores determinantes do envelhecimento saudável em idosos residentes em centro urbano: projeto epidoso. Cadernos de Saúde Pública, São Paulo, v. 19, n. 3, p. 793-798, 2003.

ROSA, T. E. C. et al. Fatores determinantes na capacidade funcional de idosos. Revista de Saúde Pública, São Paulo, v. 37, n. 1, p. 40-48, 2003.

SILVA, T. A. A. et al. Sarcopenia associada ao envelhecimento: aspectos etiológicos e opções terapêuticas. Brasil Reumatologia, São Paulo, v. 46, n. 6, p. 391-397, 2006.

SULLIVAN, S. B. O.; SCHMITZ, T. J. Fisioterapia: avaliação e tratamento. 4. ed. São Paulo: Manole, 2004.

VERAS, R. P. Gestão contemporânea em saúde: terceira idade. Rio de Janeiro: Relume Dumará, 2002. 